\title{
Fate of Bisphenols During Conventional Wastewater Treatment
}

\author{
Vehar $\mathbf{A}^{1,2}$, Kovačič $\mathbf{A}^{1}$, Hvala $\mathbf{N}^{3}$, Škufca $\mathbf{D}^{1,2}$, Levstek $\mathbf{M}^{4}$, Stražar $\mathbf{M}^{4}$, Žgajnar Gotvajn $\mathbf{A}^{5}$, Heath $\mathrm{E}^{1,2, *}$
}

1. Department of Environmental Sciences, Jožef Stefan Institute, Ljubljana, Slovenia

2. Jožef Stefan International Postgraduate School, Ljubljana, Slovenia

3. Department of Systems and Control, Jožef Stefan Institute, Ljubljana, Slovenia

4. Javno Podjetje Centralna čistilna naprava Domžale-Kamnik d.o.o., Domžale, Slovenia

5. University of Ljubljana, Faculty of Chemistry and Chemical Technology, Ljubljana, Slovenia

* Correspondence: Ester Heath; ester.heath@ijs.si

Citation: Vehar A, Kovačič A, Hvala N, Škufca D, Levstek M, Stražar M, Žgajnar Gotvajn A, Heath E. Fate of Bisphenols During Conventional Wastewater Treatment. Proceedings of Socratic Lectures. 2021; 6: 57-62. https://doi.org/10.55295/PSL.2021.D. 008

Publisher's Note: UL ZF stays neutral with regard to jurisdictional claims in published maps and institutional affiliations.

\section{(c) (1)}

Copyright: (c) 2021 by the authors. Submitted for possible open access publication under the terms and conditions of the Creative Commons Attribution (CC BY) license (https://creativecommons.org/license s/by/4.0/).

\begin{abstract}
:
There is limited knowledge of the fate of bisphenols (BPs) during wastewater treatment and their emissions into the environment via effluent release or sludge disposal. In this study, BPA and its 15 substitutes in a municipal wastewater treatment plant were monitored. First, an analytical method for determining 16 BPs in the solid phase of activated sludge, based on solid-phase extraction and gas chromatography-mass spectrometry, was developed and validated. The method was then used to analyse composite samples from different compartments of a municipal wastewater treatment plant with sequencing batch reactor technology. BPs concentrations were then converted into mass flows and their removal from wastewater and adsorption to primary and secondary sludge was determined. On average, $12 \%$ of the BPs were adsorbed to primary sludge, $2 \%$ adsorbed to secondary sludge, $18 \%$ remained in the wastewater treatment plant effluent, and $68 \%$ were removed. In addition, their emissions into the environment were also evaluated and were $2 \mathrm{~g} \mathrm{day}^{-1}$ of BPs via effluent release and $6 \mathrm{~g}_{\text {day }}{ }^{-1}$ via sludge disposal. The data shows that the emissions of BPs are not negligible, and for that reason, they should be monitored and considered.
\end{abstract}

Keywords: Bisphenol; Sludge; Wastewater; Mass balance 


\section{Introduction}

Bisphenols (BPs) are synthetic organic compounds used in the production of epoxy resins and polycarbonate, commonly, for example, in food contact materials, compact discs, construction materials, thermal paper, dental composites, medical equipment, water pipes, toys, sports equipment and dyes for synthetic clothes (Česen et al. 2018, Hu et al., 2019, Kovačič et al., 2019, Noszczyńska et al., 2018). BPA, the most common bisphenol, is a known endocrine-disrupting compound. The concern over the safety of BPA has resulted in its gradual replacement by other BPs. Since they all share a common structure of two hydroxyphenyl functionalities, the concern is that they may also share endocrine-disrupting potential (Kovačič et al., 2019).

Bisphenols can be commonly determined in surface- and groundwater, sea, sediments, soil and even dust and range from few ng L-1 or ng g ${ }^{-1}$ to few $\mu \mathrm{g} \mathrm{L}^{-1}$ to $\mu \mathrm{g} \mathrm{g}^{-1}$. The primary source of BPs to the environment are wastewaters (WW), mainly from industrial effluents and, on a smaller scale, also municipal WW (Hu et al., 2019). BPs can enter municipal WW by, e.g., food contact material leaching and synthetic clothes washing, while they enter the industrial WW by, e.g., food processing industry, food contact material industry and dry-cleaners effluents. These wastewaters are treated at the municipal wastewater treatment plants (WWTP) and BPs are, according to their physicochemical parameters, removed from the aqueous phase to different extent: some are degraded or mineralised, some remain in the aqueous phase and are discharged with effluents to surface waters, while some get adsorbed to sludge, which can be used in land applications, landfilled or incinerated.

In order to fill some gaps regarding the behaviour of BPs in WWTP, this study has the following four aims: 1) development of the method for determining 16 BPs in sludge from WW treatment, 2) determination of the concentrations of BPs in real samples of WW and sludge at different points of WWTP, 3) determination of the adsorption of BPs onto the primary and secondary sludge, their removal from the WW and their removal during the anaerobic digestion of sludge, 4) evaluation of the emissions of BPs into the environment via effluent release or sludge disposal.

\section{Methods}

An analytical method for determining 16 BPs in sludge was developed and validated. First, sludge was sampled and prepared using centrifugation and lyophilization. BPs were then extracted from the sludge to the solvent using an ultrasonic bath and centrifugation. After that, solid-phase extraction (SPE) was performed, the eluate was dried and derivatized. Samples were then analysed using gas chromatography coupled with mass spectrometry.

Aqueous samples were prepared following the method of Kovačič et al. (2019). First, aqueous samples were filtered and then loaded into SPE cartridges. The eluate was dried, derivatized and analysed after the same procedure as sludge samples.

During the development of an analytical method for sludge, two types of SPE cartridges were tested: a) OASIS Prime HLB cartridges, based on water-wettable hydrophilic-lipophilic balanced copolymer and b) Affinimip ${ }^{\circledR}$ SPE Bisphenols cartridges, based on molecularly imprinted polymer, designed for the extraction of BPs. During the optimization of the method, recovery and repeatability were monitored. In case of Oasis Prime HLB cartridges different parameters were tested: 1) five extraction solvents, 2) two centrifugation parameters and two or three extraction repetitions, 3) extract cleaning with QuEChERS and filtration, 4) influence of acidification prior to loading, 5) five washing solutions and 6) five different elution solvents. In case of Affinimip ${ }^{\circledR}$ SPE Bisphenol cartridges, two protocols with small variations suggested by a manufacturer were tested. In the end, the method using Oasis Prime HLB cartridges was chosen, since it provided the highest recoveries and repeatability, while it was also cost and time effective. Finally, the 
method performance was assessed regarding recovery, linearity, accuracy, the limit of detection and quantification, sensitivity, precision (method and instrumental repeatability) and the matrix effect.

WW and sludge sampling was performed at the Central Wastewater Treatment Plant Domžale-Kamnik, which has a capacity of 149,000 population equivalents. 24-hour composite samples of influent to the WWTP (WWTP_inf), influent to the primary settler (PSE_inf), effluent from the primary settler (PSE_eff) and with 24-hour delay effluent from the WWTP (WWTP_eff) were sampled. 6-hour composite samples of primary (PS) and secondary sludge (SS) were sampled, and one grab sample of anaerobically stabilised sludge (AS) was sampled on the same day (Figure 1).

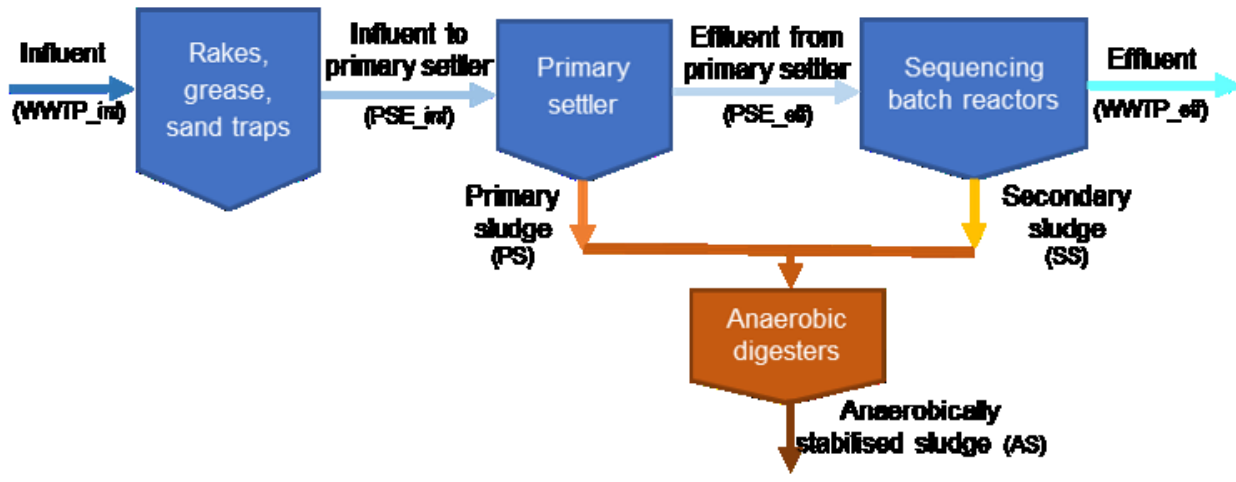

Figure 1. Scheme of a WWTP and the flows where samples were taken

\section{Results}

In the WW flows, BPA and BPS were the most abundant and 22BPF, 24BPF, 44BPF and BPE to a lesser extent (Figure 2). The concentration of BPs increased during the mechanical stage with the highest values in PSE_eff. All BPs in the WWTP_eff were below the limit of quantification (LOQ(BPA) $=18 \mathrm{ng} \mathrm{L}^{-1}$, LOQ $\left.(15 \mathrm{BPs})=2 \mathrm{ng} \mathrm{L}^{-1}\right)$ except BPA (79 $\left.n g \mathrm{~L}^{-1}\right), \mathrm{BPS}\left(20 \mathrm{ng} \mathrm{L^{-1 }}\right)$, BPBP $\left(3 \mathrm{ng} \mathrm{L}^{-1}\right), 22 \mathrm{BPF}\left(3 \mathrm{ng} \mathrm{L}^{-1}\right)$ and BPAP $\left(2 \mathrm{ng} \mathrm{L}^{-1}\right)$.

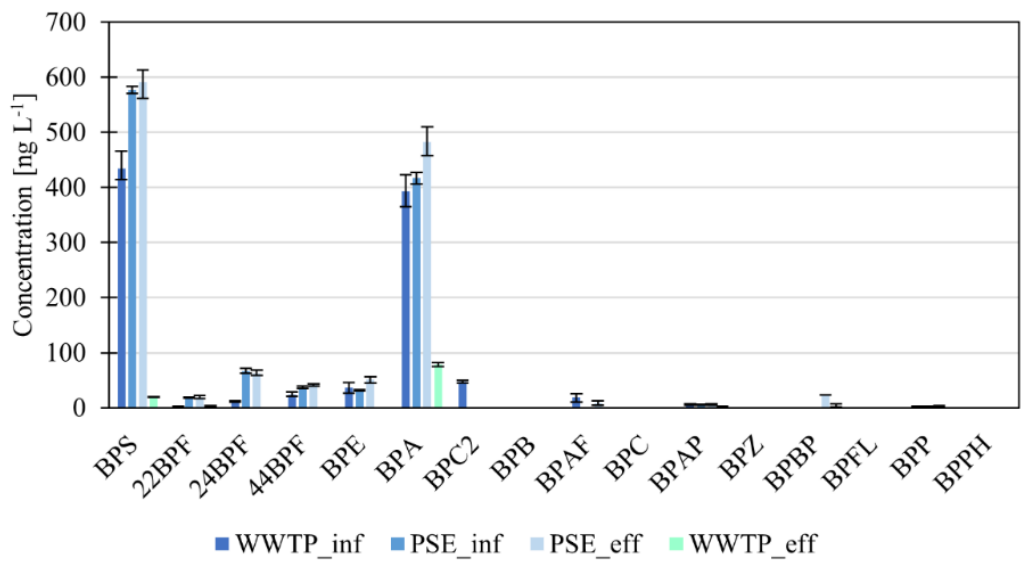

Figure 2. The concentrations of BPs in the WWTP_inf, PSE_inf, PSE_eff, WWTP_eff

In the sludge, the most abundant BPs were BPA and BPS, while 22BPF, 24BPF and $44 \mathrm{BPF}$ were present to a lesser extent (Figure 3). The highest concentrations of BPs were observed in the anaerobically stabilised sludge, compared to the primary and secondary sludge, where BPA reached $946 \mathrm{ng} \mathrm{g}^{-1}$ in the solid phase (SP) and $3248 \mathrm{ng} \mathrm{L}^{-1}$ in the aqueous phase (AP). 

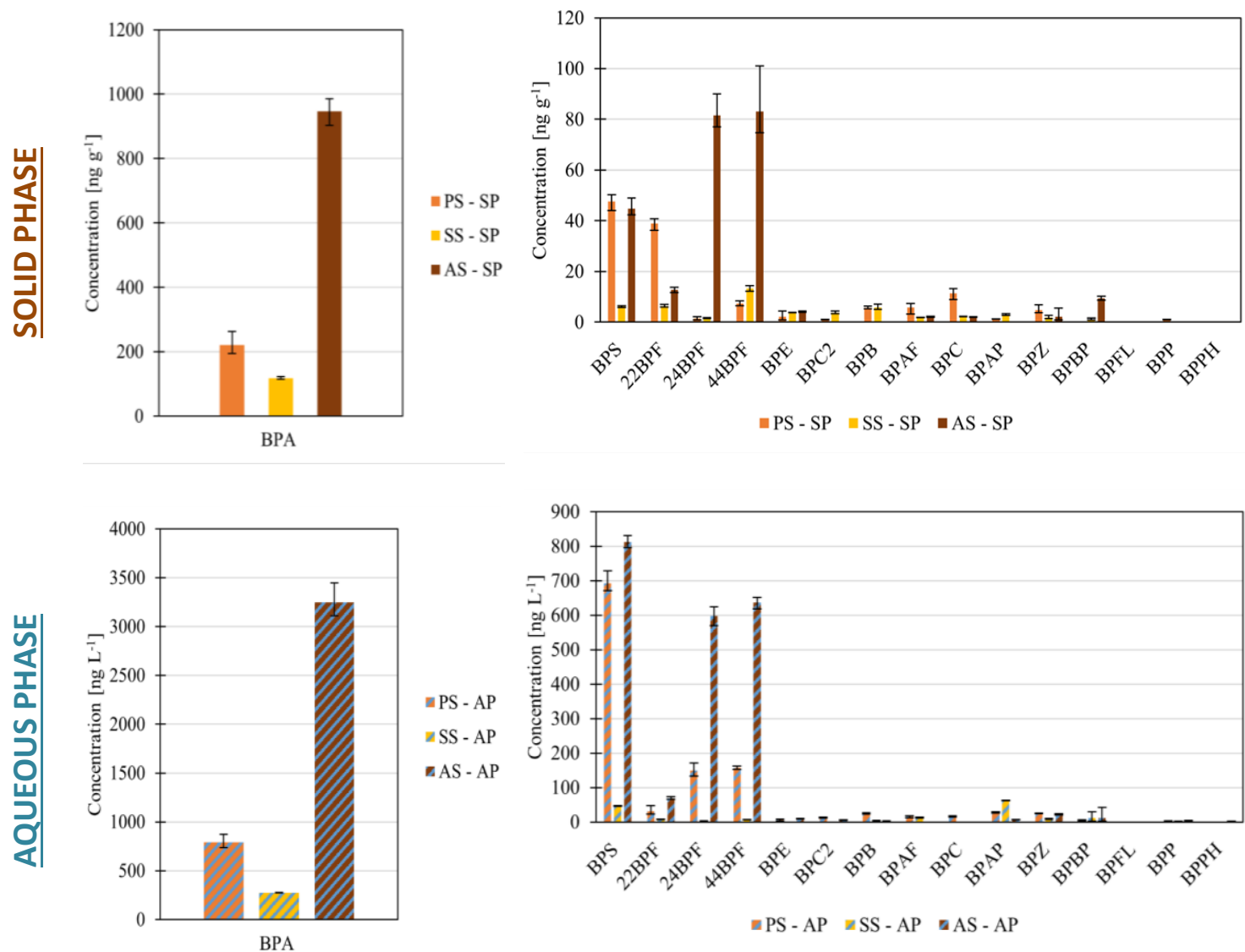

Figure 3. Levels of BPs in the solid (SP) and aqueous phase (AP) of primary (PS), secondary (SS), and anaerobically stabilised sludge (AS)

According to the calculated mass flows of BPs (Figure 4), BPA and BPS were again the most abundant in wastewater. The trend of mass flows rising through the mechanical stage of treatment with the maximum in the PSE_eff and the trend of maximal mass of BPs flows in the AS, lower in PS and the lowest in SS were present again.

Possible explanations for the increasing concentrations of BPs through the mechanical stage of treatment can be adsorption and later desorption of the compounds at different parts of the mechanical stage of WWTP. Besides, the concentrations of BPs in the solid particles in WW flows were not measured, since the content of solid particles is so low that is usually not determined. There are three possible reasons for the higher concentrations of BPs in the influent to the primary settler. The first could be receiving the external WW (septic tanks), which are delivered by trucks. The second could be the inflow of the wastewater from the deamonification process of the centrate, which comes from the mechanical thickening of the anaerobically stabilised sludge. The third reason could be the inflow of pretreated wastewater, which is a result of the treatment of hazardous and non-hazardous liquid waste in the electrocoagulation plant. All the above-mentioned types of WW go directly to the mechanical stage of WWTP.

One of the possible reasons for the higher amounts of BPs in the anaerobically stabilised sludge is its single grab sampling. Since its retention time is 30 days, determined concentrations cannot be directly related to the concentrations of BPs in primary and secondary sludge. The other reasons can be adsorption and later desorption of the compounds at the parts of the anaerobic reactor, deconjugation of conjugated compounds as well as addition of external sludge, going directly to anaerobic stabilisation. 


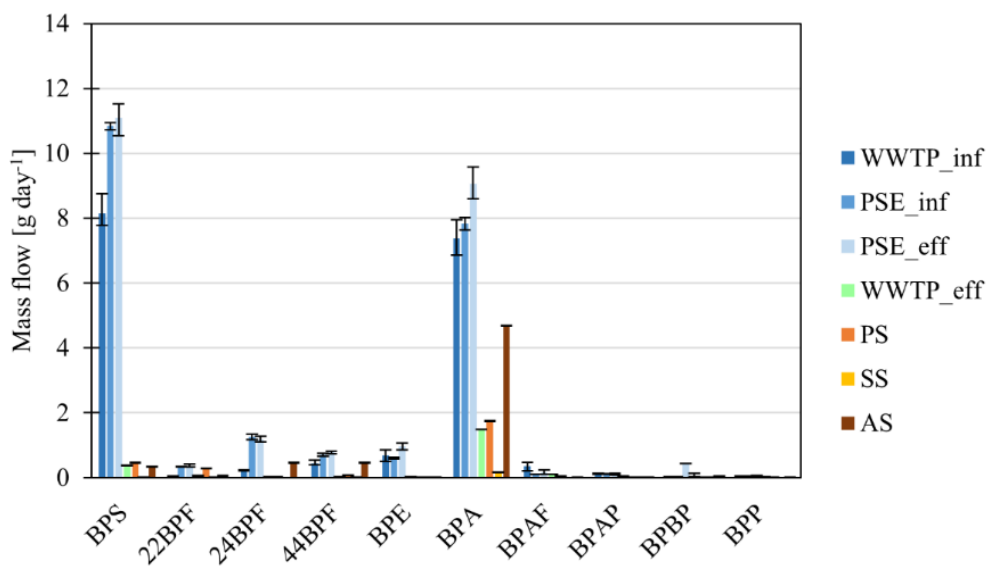

Figure 4. Mass flows of BPs in the selected wastewater flows and sludge

On average, $12 \%$ of the BPs were adsorbed to PS, $2 \%$ adsorbed to SS, $18 \%$ remained in the WWTP_eff, and 68\% were removed (Figure 5). The lowest removal efficiency $(29 \%)$ was observed for BPAF, a halogenated bisphenol, which is in agreement with the fact that compounds with strong C-F bonds are generally poorly biodegradable under aerobic conditions (Kovačič et al., 2019). The second-lowest removal efficiency was obtained for BPP, where the mass flows through the treatment system were relatively low. In case the concentrations were below the LOQ, the removal was calculated from the LOQ values.

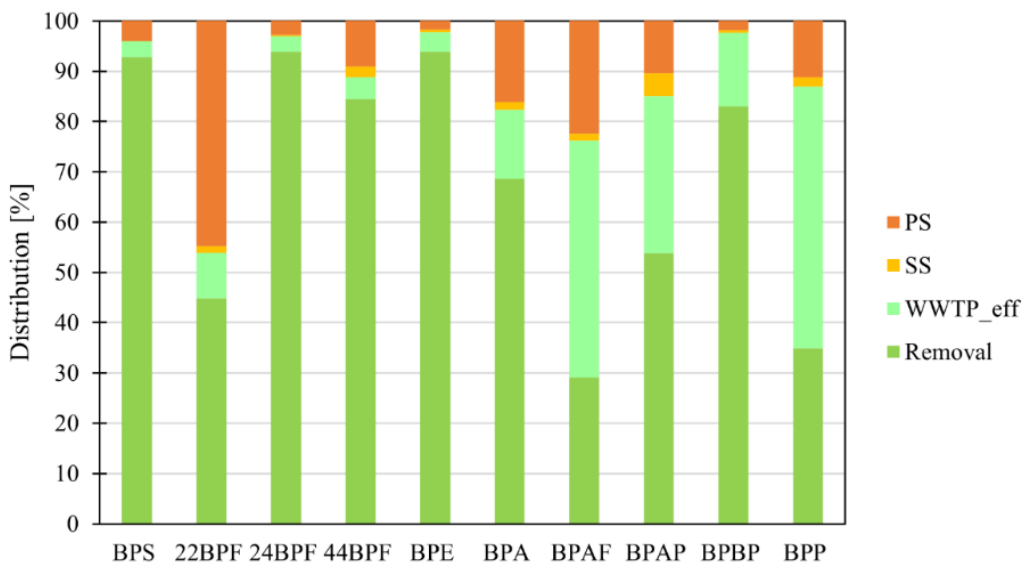

Figure 5. Distribution of BPs in primary (PS) and secondary sludge (SS), WWTP_eff and their removal (\%)

Removal of BPs in anaerobic conditions was successful only for 6 BPs (Figure 6, A), on average $52 \%$. The negative removal in anaerobic conditions was shown for 4 BPs (Figure 6, B), which means that the mass flows of BPs in sludge increased after the anaerobic digestion. The anaerobic removal of BPAF was the highest $(79 \%)$ among all BPs, which was expected since halogenated compounds are known to be more biodegradable in anaerobic conditions. 

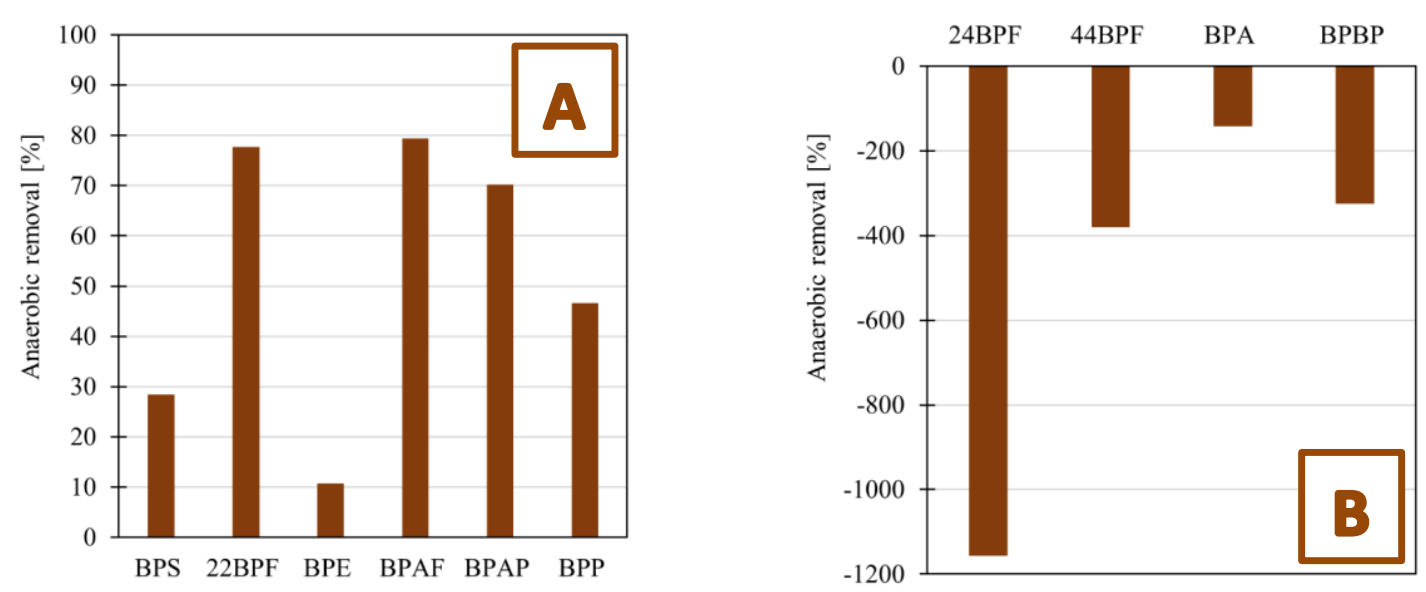

Figure 6. Removal of BPs in anaerobic digesters: A) anaerobic removal (\%) for BPS, 22BPF, BPE, BPAF, BPAP, BPP and B) negative anaerobic removal (\%) for 24BPF, 44BPF, BPA, BPBP

\section{Conclusions}

An analytical method for determining 16 BPs was developed and the concentrations of BPs at different points in WWTP were determined. The majority of BPs were removed during the wastewater treatment, while the others remained in the effluent or were adsorbed to anaerobically stabilised sludge. In case of investigated WWTP, each day $2 \mathrm{~g}$ of BPs end up in the receiving river through effluent discharge and $6 \mathrm{~g}$ are emitted through anaerobically stabilised sludge disposal. Even though the concentrations of BPs in the effluent are low, there are also many other contaminants of emerging concerns present in WW effluents, and since their long-term effects are still very poorly researched, the impacts of the effluent discharges on the environment, river organisms and humans are still to be researched and the inclusion of advanced wastewater treatment techniques, as pre- or post-treatment may be a solution, which would lead to mineralization of organic contaminants to $\mathrm{CO}_{2}$ and $\mathrm{H}_{2} \mathrm{O}$. Before taking a final decision on the potential of sludge for agricultural or remediation application, the presence of other hazardous contaminants like drugs, heavy metals and their residues has to be evaluated and risk assessment performed. Despite the high costs, currently, the most suitable sludge management option is still incineration.

Funding: This research was supported by the Slovenian Research Agency ARRS, namely Research Program Cycling of Substances in the Environment, Mass Balances, Modelling of Environmental Processes and Risk Assessment P1-0143 (projects N1-0143, L7-1848, J1-9166), Research Program Chemical Engineering P2-0191 and Research Program Systems and Control P2-0001.

Conflicts of Interest: The authors declare no conflict of interest.

\section{References}

1. Česen M, Lenarčič K, Mislej V, et. al. The occurrence and source identification of bisphenols compounds in wastewaters. STOTEN. 2018; 616-617: 744-752. DOI: 10.1016/j.scitotenv.2017.10.252

2. $\mathrm{Hu}$ Y, Zhu Q, Yan X, Liao C, Jiang G, Occurrence, fate and risk assessment of BPA and its substituents in wastewater treatment plant: A review. Environmental Research. 2019; 178: 108732. DOI: 10.1016/j.envres.2019.108732

3. Kovačič A, Česen M, Laimou-Geraniou M, et. al. Stability, biological treatment and UV photolysis of 18 bisphenols under laboratory conditions. Environmental Research. 2019; 179: 108738. DOI: 10.1016/j.envres.2019.108738

4. Noszczyńska M, Piotrowska-Seget Z, Bisphenols: Application, occurrence, safety, and biodegradation mediated by bacterial communities in wastewater treatment plants and rivers. Chemosphere. 2018; 201: 214-223. DOI:

10.1016/j.chemosphere.2018.02.179 